\title{
35. TIPIFICACIÓN DE HYOSERIS MINIMA L. (ASTERACEAE)
}

Salvador TALAVERA y María TALAVERA*

Recibido el 1 de octubrede 2015, aceptado para su publicación el 19 de octubre de 2015

Typification of Hyoseris minima L. (Compositae)

Palabras clave: Arnoseris, lectótipo, Linnaeus, Cichoriinae.

Key words. Arnoseris, lectotype, Linnaeus, Cichoriinae.

Arnoseris Gaertn. es un género propio de Eurasia, aunque recientemente se ha encontrado Arnoseris minima (L.) Schweigg. \& Körte, su única especie, cerca de Baberred, en las montañas del Rif, NW de Marruecos (Valdés, 2013: 93). Estudios recientes de filogenia molecular han puesto de manifiesto que Arnoseris es el hermano filogenético de Tolpis Adans. (Gruenstaeudl \& al. 2013), género que comprende c. 16 especies distribuidas por el W de la Región Mediterránea y por los cuatro archipiélagos de la Región Macaronésica, en donde sufrió una gran radiación adaptativa, sobre todo en el archipiélago de las Canarias.

En el presente trabajo, se aborda la lectotipificación de Hyoseris minima L., el basiónimo de Arnoseris minima.

Hyoseris minima L., Sp. Pl.: 809 (1753) [三 Arnoseris minima (L.) Schweigg. \& Körte, Fl. Erlang. 2: 72 (1811)]

三Lapsana gracilis Lam., Fl. Franç. 2: 102

(1779), nom. illeg.

$\equiv$ Lapsana minima (L.) All., Fl. Pedem. 1: 206 (1785)

$\equiv$ Arnoseris pusilla Gaertn., Fruct. Sem. Pl.

2: 355 (1791), nom. illeg.

$\equiv$ Hyoseris exigua Salisb., Prodr. Stirp.
Chap. Allerton: 182 (1796), nom. illeg. $\equiv$ Lapsana pusilla (Gaertn.) Willd., Sp. Pl. 3: 1623 (1804), nom. illeg.

三Arnoseris clavata Bubani, Fl. Pyren. 2: 49 (1899), nom. illeg.

$\equiv$ Cichorium arnoseris E.H.L. Krause, Deutschl. Fl. (Sturm.), ed. 2, 14: 180 (1906), nom. illeg.

Ind. loc.: "Habitat in Europae arvis apricis" Lectótipo (designado aquí): Herb. Linn. No. 957.6 (LINN)

Cuando Linneo (1753: 809) describió Hyoseris minima, indicó: "5. Hyoseris minima caule diviso nudo. Hort. Cliff. 386. Fl. Suec. 630. Roy. Lugdb. 127. Sauv. monsp. 14. Hyoseris angustifolia. Tabern. ic. 180. Hieracium minimum. Clus. hist. 2. p. 142".

De los seis antecedentes que aporta Linneo y sirven para la elección del tipo de Hyoseris minima L., las referencias del propio Linneo (1738: 386; 1745: 227, n 630), así como las de van Royen (1740: 127) y Sauvages (1751: 14), coinciden en el mismo nombre frase: "Hyoseris caule diviso nudo". Las otras referencias se corresponden con icones, uno en la obra de Tabernamontanus (1590: 180, Fig. Hyoseris

Este trabajo ha sido financiado por el proyecto Flora iberica (CGL2012-32914) y cofinanciado con fondos FEDER 
angustifolia), y el otro en la de Clusius (1601, 2: tab. CXLII, Hieracium IX minimum). Para la preparación de la primera edición de su Species Plantarum, los únicos materiales que Linneo pudo utilizar para la descripción de Hyoseris minima fueron los de su herbario o el de Adriaan van Royen, depositado en la actualidad en el Rijksherbarium de Leiden, Holanda (L).

Como indicó Jarvis (2007: 581), existen dos pliegos que contienen materiales originales, uno en el herbario principal de Linneo, en la Sociedad Linneana de Londres (LINN no. 957.6), y otro en el herbario de van Royen (L no. 900.74-407). El pliego depositado en el herbario de Linneo (LINN no. 957.6) contiene una planta completa, de unos $30 \mathrm{~cm}$, con los tallos divididos y sin hojas, que coincide plenamente con la diagnosis de Linneo y que se corresponde con lo que se conoce como Arnoseris minima. Bajo este ejemplar Linneo anotó: "5. minima", el doble código que utilizó en la $1^{\mathrm{a}}$ edición del Species Plantarum para nombrar las especies dentro de un género. El pliego que se encuentra en el herbario de A. van Royen contiene también una planta completa, de c. $15 \mathrm{~cm}$, muy parecida a la anteriormente comentada del herbario de Linneo.

La planta representada en el icón de la obra de Tabernamontanus no se puede identificar como perteneciente a Hyoseris minima, pues su raíz es gruesa, las hojas son lineares (uno de los tallos tiene una hoja bien desarrollada) y los pedúnculos de los capítulos son completamente cilíndricos, y no se ensanchan en el ápice como es característico en Hyoseris minima. Por el contrario, el icón de la obra de Clusius representa fielmente una planta de Arnoseris minima.

De los tres elementos que disponibles para la elección del tipo, se elige como lectótipo el ejemplar que se encuentra en el herbario de la Sociedad Linneana de Londres (LINN 957.6) por ser, de los tres, del que se tiene constancia fue utilizado por Linneo cuando describió su especie.

\section{BIBLIOGRAFÍA}

CLUSIUS, C. -1601- Rariorum Plantarum Historia, Lib. 5. Amberes (Bélgica).

GRUENSTEUDL, M., SANTOS-GUERRA, A. \& R. JANSEN -2013- Phylogenetic analyses of Tolpis Adans. (Asteraceae) reveal patterns of adaptive radiation, multiple colonization and interspecific hybridization. Cladistics 29: 416-434

JARVIS, C. - 2007- Order out Chaos. Linnean Society of London: Londres.

LINNEO, C. -1738-Hortus Cliffortianus. Amsterdam.

LINNEO, C. -1745-Flora Suecica. Stockholmiae. LINNEO, C. -1753- Species Plantarum. Holmiae.

ROYEN, A. VAN -1740- Flora Leidensis Prodromus. Lugduni Batavorum, Academiae Typographum.

SAUVAGES, F.B. -1751- Methodus foliorum seu Plantae Florae Monspeliensis. La Haya.

TABERNAMONTANUS, T.J. -1590- Eicones Plantarum. Francofurti [Fráncfort de Mena (Alemania)].

VALDÉS, B. -2013- Results of the fifth "Iter Mediterraneum" in Morocco, 8-27 June, 1992. Bocconea 26: 5-12, 13-132.

Dirección de los autores. Departamento de Biología Vegetal y Ecología (Botánica). Facultad de Biología, apartado 1095.41080-Sevilla.mtalavera@us.es 